\title{
Neural generators of psychogenic seizures: Evidence from intracranial and extracranial brain recordings
}

\author{
Shahar Arzy a,b,*, Pär Halje ${ }^{\text {c,e }}$, Daniel S. Schechter ${ }^{\text {d }}$, Laurent Spinelli b ${ }^{\text {, Margitta Seeck }}{ }^{\mathrm{b}}$, Olaf Blanke ${ }^{\mathrm{b}, \mathrm{c}}$ \\ a Department of Neurology, Hadassah Hebrew University Medical Center, Jerusalem, Israel \\ b Department of Neurology, University Hospital, Geneva, Switzerland \\ c Laboratory of Cognitive Neuroscience, Center for Neuroprosthetics \&' Brain Mind Institute, School of Life Sciences, Ecole Polytechnique Fédérale de Lausanne, Lausanne, Switzerland \\ d Department of Child Psychiatry, University Hospital, Geneva, Switzerland \\ e Faculty of Experimental Medical Sciences, Lund University, Sweden
}

\section{A R T I C L E I N F O}

\section{Article history:}

Received 5 October 2013

Accepted 13 October 2013

Available online xxxx

\section{Keywords:}

Psychogenic seizures

Frequency analysis

Intracranial recording

Theta rhythm

EEG

\begin{abstract}
A B S T R A C T
Psychogenic seizures (PSs) convincingly mimic seizure phenomena but with no underlying epileptic activity. However, not much is known about their neurophysiological basis. We had the rare opportunity to analyze intracranial brain recordings of PSs occurring besides epileptic seizures (ESs), which identified distinct frequency changes over the parietal cortex. For further validation, we applied topographic frequency analysis to two other patients who presented PSs and ESs during long-term monitoring. The analysis revealed a power decrease in the theta band at the posterior parietal cortex in all three patients during PSs but not during ESs. These changes may reflect disturbed self-referential processing associated with some PSs.
\end{abstract}

(c) 2013 Elsevier Inc. All rights reserved.

\section{Introduction}

Psychogenic seizures (PSs) embrace an extensive range of paroxysmal episodes of impaired self-control associated with a range of motor, sensory, autonomic, and mental manifestations, similar to that of epileptic disorders, yet without underlying epileptic activity [1-3]. Psychogenic seizures may appear in patients with epilepsy in addition to epileptic seizures (ESs) and in otherwise healthy individuals. However, PSs in both groups are usually induced by psychosocial stressors [4]. Not much is known about PS's neural basis, as general medical and neurological assessments, including electrophysiological and neuroimaging studies, are unremarkable. We hypothesized that signal analysis of PSs would show subtle differences between PS, ES, and nonseizure (NS) states. Based on the relations of PSs to selfrelated stressors, we speculated that functional changes would be found in brain regions related to self-referential activity and/or retrieval of old memories of stressogenic events. These processes might translate to underlying neurophysiological changes, which in the case of PSs ultimately evolve into abrupt behavioral changes. Few recent studies using resting state fMRI (RSfMRI) have found functional changes in PSs. Ding and colleagues [5] found changes in small-world property

\footnotetext{
* Corresponding author at: Neuropsychiatry Lab, Department of Neurology, Hadassah Hebrew University Medical Center, Israel. Fax: +972 26437782.

E-mail address: shahar.arzy@ekmd.huji.ac.il (S. Arzy).

URL: http://www.mind.huji.ac.il (S. Arzy).
}

(functional connectivity) of the RSfMRI recordings in 11 patients. Van der Kruijs and colleagues found stronger connectivity values between regions involved in emotion (insula), executive control (inferior-frontal and parietal cortices), and movement (precentral sulcus) [6]. Using voxel-based morphometry, Labate and colleagues [7] found abnormal cortical atrophy of the motor and premotor regions in the right hemisphere and the cerebellum bilaterally. Using EEG, Krishnan et al. found "resetting" of brain dynamics in patients with ESs but not in patients with PSs [8]. In the present study, we had the rare opportunity to analyze multichannel intracranial EEG data from a patient with PSs and ESs, as well as extracranial data from two other patients with combined PSs and ESs, in a quest for identifying in brain activity related to PSs.

\section{Materials and methods}

\subsection{Clinical examinations}

All patients with PSs and ESs who were evaluated in the presurgical epilepsy unit at the Department of Neurology in Geneva University Hospital during the study time were included. Comprehensive neurological examination, neuropsychological tests, video-monitored EEG, and structural MRI were done for each patient. Detailed psychiatric evaluation was performed in order to identify possible conflicts that may underlie PSs. Patients gave informed consent for the investigations and were rendered anonymous for this study, which was approved by the ethical committee of Geneva University Hospital. 


\subsection{Electrophysiology}

Continuous video-EEG was recorded using 120 intracranial electrodes for patient 1 (Fig. 1A) (1024-Hz digitization) and 32 surface EEG electrodes for patients 2-3 (Deltamed $\AA$, France; impedances, $<10 \mathrm{M} \Omega$; vertex referenced; $512-\mathrm{Hz}$ digitization; band-pass filtered, $0.1-1500 \mathrm{~Hz}$ ). Twelve ictal EEG epochs of 5 seconds each were extracted from periods in which patients had their habitual semiology (1-2 EEG epochs for each paroxysm). Interictal (nonseizure, NS) epochs were extracted while patients had no ictal manifestations. Periods were extracted when patients were free of medications. During these periods, patients were lying still with their eyes closed. Preprocessing included the removal of $50 \mathrm{~Hz}$ from the raw signals using a notch filter offline. For the intracranial recorded data, the 5-second nonreferential time epochs were recomputed against the averaged reference and then transformed into the frequency domain using fast Fourier transform (FFT). This was done using the Hartley algorithm and a Hanning window within the range of $0-80 \mathrm{~Hz}$ in $0.5-\mathrm{Hz}$ steps. The power for each frequency band was averaged over the extracted epochs. Statistical analysis (unpaired t-test, $\alpha<0.01$, two-tailed after Bonferroni corrections) was run on the average power of each frequency band, comparing the global power distribution along frequencies between ESs, PSs, and NSs. For the extracranially recorded data (patients 2-3), a frequency analysis was applied on the conventional frequency bands of $\delta$ (1-4 Hz), $\theta(4-8 \mathrm{~Hz}), \alpha(8-13 \mathrm{~Hz}), \beta_{1}(13-18 \mathrm{~Hz}), \beta_{2}(18-$ $24 \mathrm{~Hz}), \beta_{3}(24-30 \mathrm{~Hz}), \gamma_{1}(30-40 \mathrm{~Hz}), \gamma_{2}(40-50 \mathrm{~Hz}), \gamma_{3}(50-60 \mathrm{~Hz})$, $\gamma_{4}(60-70 \mathrm{~Hz})$, and $\gamma_{5}(70-80 \mathrm{~Hz})$. The resulting complex values (sine and cosine coefficients) were subjected to the "FFT approximation procedure" $[9,10]$. This method transforms the FFT-analyzed multichannel data into phase-corrected potential distribution maps within a certain frequency band. For each patient, potential maps were subjected to topographic ANOVA (TANOVA) tests ( $\alpha<0.05$ after multiple permutation correction). Alias TANOVA is a nonparametric/ randomization test in which the variable tested is the dissimilarity (the square root of the mean of the squared differences between the potentials measured at all corresponding electrodes) between conditions (PSs, ESs, and NSs), showing the topographical change without accounting for intensity. The potential maps obtained were averaged over the epochs separately for each frequency band and for each subject. A permutation procedure was applied in order to control for the arbitrariness of the maps' polarity [9,10]. Resulting from these procedures were potential maps showing differences in brain topography (and, therefore, neural generators) in specific frequency bands characterizing the topographic frequency differences between ictal and interictal states for each patient. Brain generators underlying the averaged potential maps were localized on the individual patient's MRI using electrical neuroimaging (LAURA). Three-dimensional reconstruction of the individual patient's MRI was performed using the SMAC toolbox and the Cartool Software.

\subsection{Linear discriminant classifier}

Fast Fourier transform analysis showed differences between ESs, PSs, and NSs to be mostly manifested at the theta and gamma bands (see below). These were further demonstrated for each single trial using Fisher linear discriminant (FLD) analysis. The Fisher linear discriminant is a linear classifier that maximizes the ratio of "between-class" variance to "within-class" variance so that the highest discrimination is achieved [11]. The Fisher linear discriminant may therefore discriminate between gamma and theta components for each condition. The Fisher linear discriminant was hence applied on the extracted Fourier features for each patient in each condition. The results were validated using the leave-one-out cross-validation method. This method consists in iteratively discarding all the data one at a time, learning from the remaining data, and classifying the discarded one. The results for all discarded data are subsequently pooled. A comparison with the result obtained by learning from the whole data set is performed thereafter. The statistical level of error was set to $\alpha<0.05$.

\section{Results}

\subsection{Intracranially recorded case}

In order to exemplify PSs, we detail here the clinical history of patient 1 , who was further investigated by intracranial recordings. Patient 1 was a 17-year-old woman with normal psychiatric history who was undergoing evaluation for surgical treatment of epilepsy. Daily ESs were characterized by loss of consciousness, staring, bilateral nystagmus, bilateral hand jerking, atonia, and falls, followed by postictal drowsiness. Two months before intracranial electrode implantation, the patient manifested a different phenomenology with prolonged loss of contact, staring, and spasm, without nystagmus, hand jerking, or postictal signs. One hundred twenty subdural electrodes covering her left cerebral hemisphere (Fig. 1A) were implanted to identify the epileptic seizure focus and language cortex for presurgical evaluation. Clinical EEG analysis of the implanted electrodes revealed an epileptic focus in the left posterior temporal cortex (Fig. 1A). No EEG changes were found during prolonged loss of contact and staring, hence, allowing the diagnosis of these events as PSs.

\subsection{Phenomenological results}

All patients were women aged 17-36 years old. Two patients ( 1 and 3 ) had a clear stressogenic event related to their epileptic disease (forthcoming operation), and one (2) was facing important conflicts in her immediate family of origin (divorce, dismissal). Psychogenic seizures in all these patients were characterized by loss of contact, spasm, and speech arrest. Psychogenic seizures were of high frequency $(>1 /$ day $)$ in all patients.

\subsection{Electrophysiological results}

To investigate a potential neurobiological basis for patient 1's PSs, we analyzed intracranial electrophysiological signals for all 120 implanted electrodes during PS, ES, and NS epochs using power-frequency and Fisher linear discriminant analyses with cross-validation procedure. These analyses detected six neighboring electrodes in the posterior parietal cortex, posterior and superior to the epileptogenic focus, that showed a significant decrease in theta band power $(4-8 \mathrm{~Hz})$ during PSs, as compared to NSs and ESs (Figs. 1B-D). Power-frequency and Fisher linear discriminant analyses of ESs showed significant gamma power increases $(>30 \mathrm{~Hz})$, maximal over the left posterior temporal cortex. Applying topographic frequency analysis and Fisher linear classifier to extracranial recordings of PSs in patients 2 and 3, we found a significant decrease in theta power in the left (patient 2) and bilateral (patient 3) posterior parietal cortex during PSs (Fig. 2). Findings were also significant for left frontocentral gamma power increases during ESs (compared to PSs and NS).

\section{Discussion}

Here, we found specific changes in brain rhythm in the theta band at the posterior parietal cortex during PSs of similar semiology in three patients, one of them recorded intracranially allowing validation by direct cortical recordings. All patients were confronted with stressogenic experiences, in concordance with previous studies suggesting stress as an important risk factor for the development of PSs. Analysis of intracranial recordings of patients with epilepsy in the resting state showed that a cluster at the theta range characterizes resting-state activity at the 'default-mode network' in which the posterior parietal cortex plays a key role [12]. This is in concordance with previous resting-state fMRI findings showing stronger connectivity values within 


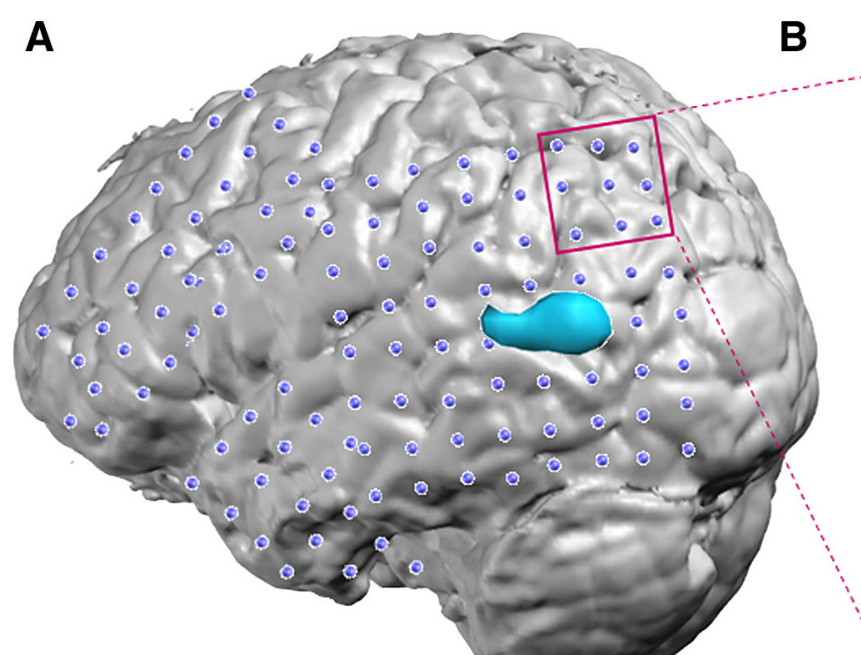

B

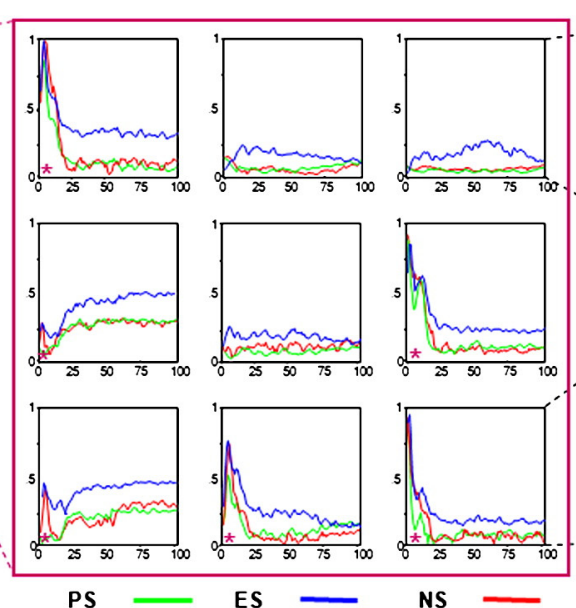

C

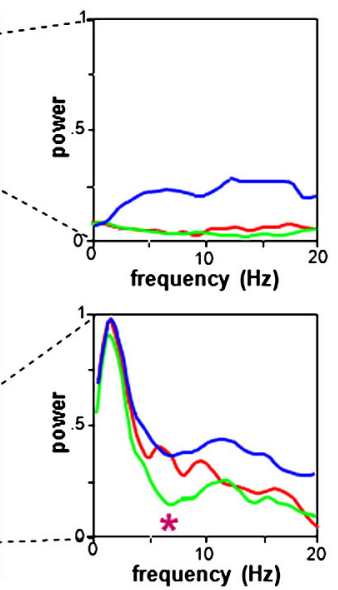

D

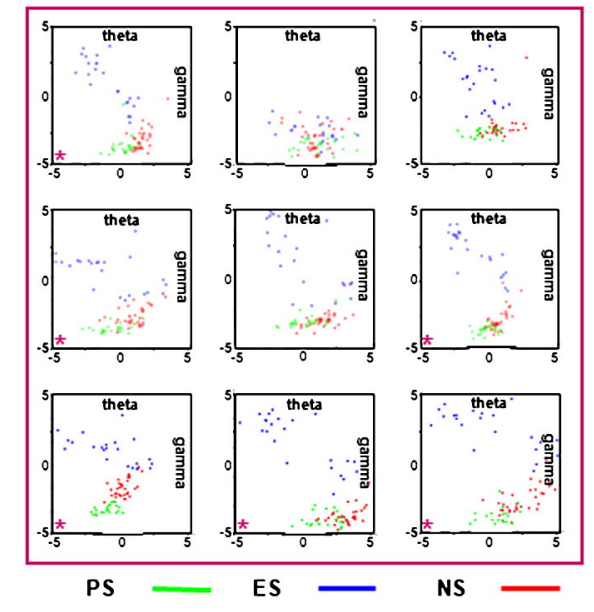

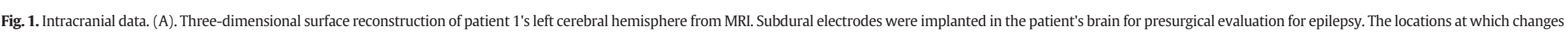

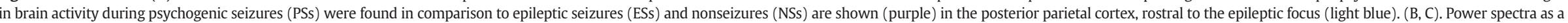

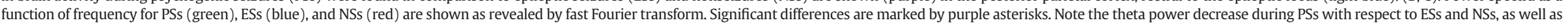

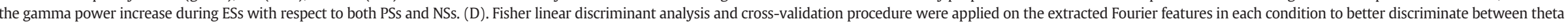
(X-axis) and gamma (Y-axis) activity during PS (green), ES (blue), and NS (red) conditions for each single trial. Note the theta power decrease during PSs and gamma power increase during ESs with respect to NSs. 
A
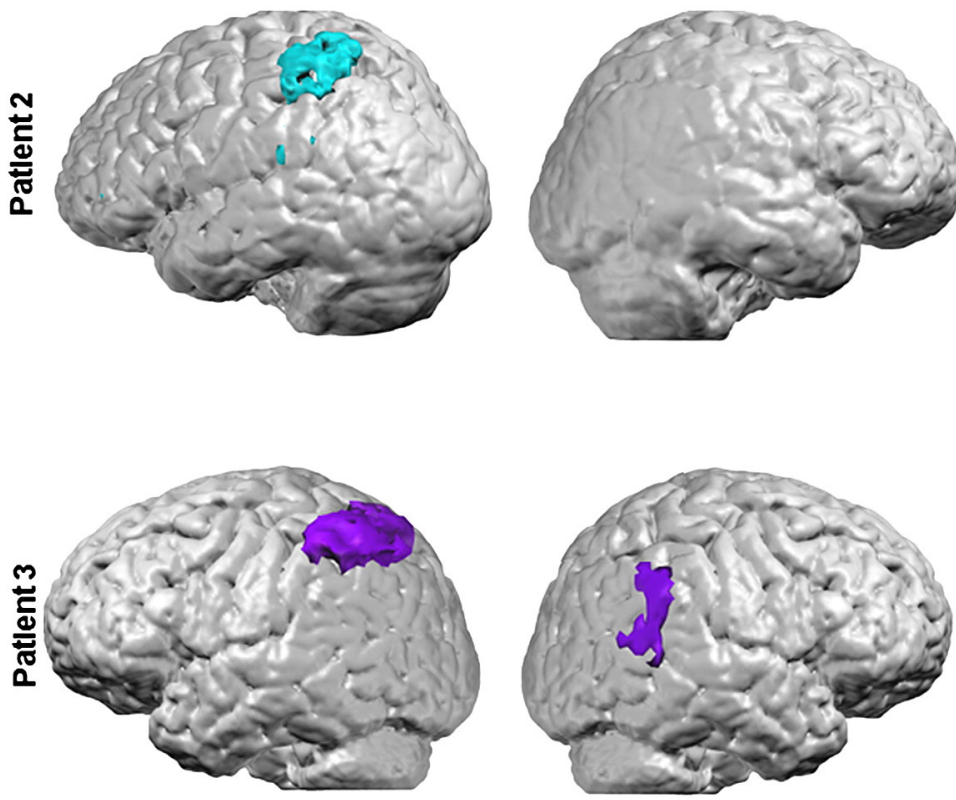

B

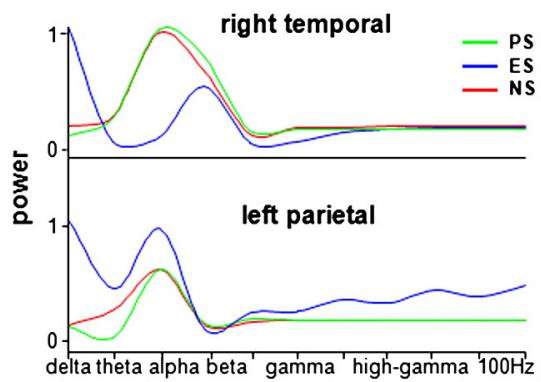

frequency bands

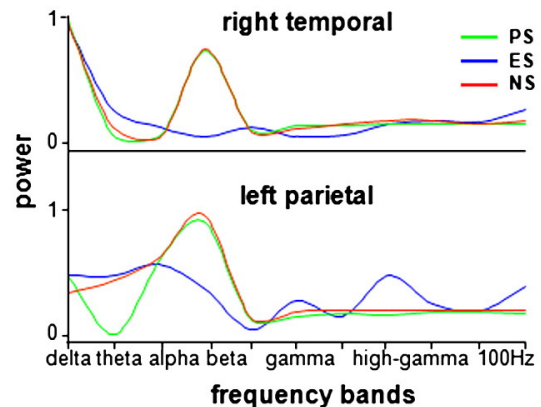

C
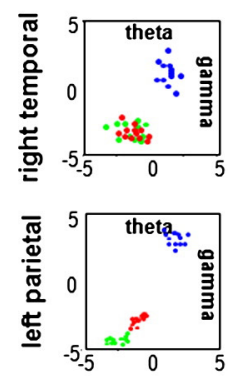

- PS

- ES
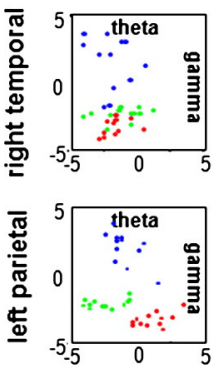

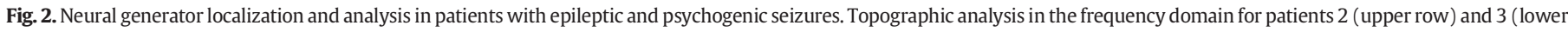

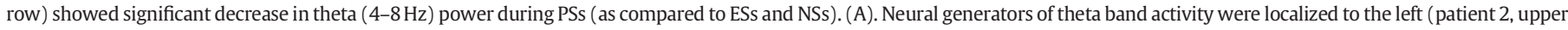

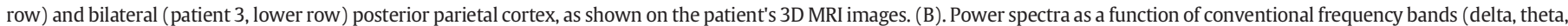

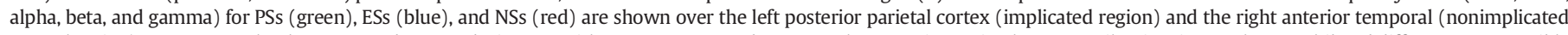

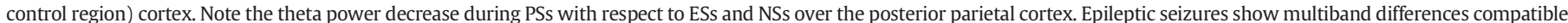

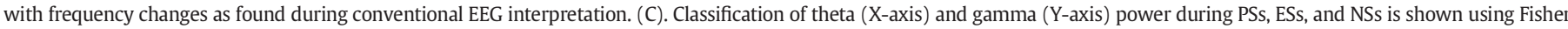

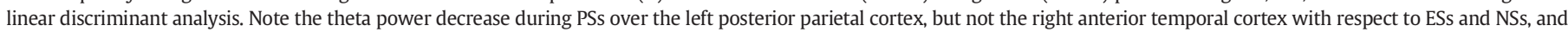
bilateral gamma power increases during ESs with respect to PSs and NSs.

major cortices of the default-mode network including the inferior frontal gyrus, the parietal cortex, and the insula in patients with PSs [6]. More specifically, theta activity is implicated in memory processes, as well as emotional arousal $[13,14]$. The posterior parietal cortex is related to self-processing [15] and is implicated in autobiographical thinking about past and future events [16]. We speculate that PSs, reflecting conflicts related to one's past and current self-referenced dilemmas, are related to a dysfunction of the default-mode network [12] as reflected here by changes in theta band activity in the posterior parietal cortex, a central hub of the default-mode network, which embodies emotional and autobiographical memory functions.

The present study is not free of limitations. First, we analyzed a small number of patients with heterogenic PS etiology. However, the similar results, which were found despite these differences, may hint on their relations to the PSs rather than to other external factors. In addition, it could be argued that EEG has limited localization ability. However, patient 1 was recorded intracranially using subdural electrodes, and similar changes as in the scalp recordings of patients 2 and 3, during similar PS semiology, were noted. Finally, the comparison with the ictal and interictal states strongly suggests that the theta changes were distinct to PSs. Further research is thus needed in patients with PSs without ESs as well as with regard to the involvement of large scale networks (such as the default-mode network, emotion-related network, or the motor network) in PSs.

In conclusion, we propose that the EEG associated with at least a subset of PSs undergoes significant neurophysiological changes, separating these epochs from the patients' habitual seizures and their interictal state. A decrease in the theta frequency band, also crucial for memory and emotional processes, is noted during PSs. Topographic analysis in the frequency domain enabled us to localize neural changes during PSs to brain generators at the posterior parietal cortex, a selfprocessing related region which may be disturbed in PSs.

\section{Disclosures}

We confirm that we have read the Journal's position on issues involved in ethical publication and affirm that this report is consistent with those guidelines. Dr. Arzy was supported by the Marie Curie IntraEuropean Fellowship within the framework of the EU-FP7 program and the Ecole Polytechnique Fédérale de Lausanne (EPFL)-Hebrew University brain collaboration. Dr. Seeck is supported by SNF grant nos. 140332 and 140338 . Dr. Blanke was supported by The Swiss National Science Foundation and the Bertarelli Foundation.

\section{Acknowledgment}

We thank Professors T. Ben-Hur (Hadassah Hebrew University Medical Center) and Peter W. Kaplan (Johns Hopkins Medical Center) for their valuable input on previous versions of the manuscript.

\section{References}

[1] Reuber M. Psychogenic nonepileptic seizures: answers and questions. Epilepsy Behav 2008;12:622-35.

[2] Brown RJ, Syed TU, Benbadis S, LaFrance WC, Reuber M. Psychogenic nonepileptic seizures. Epilepsy Behav 2011;22:85-93.

[3] LaFrance WC. Psychogenic nonepileptic seizures. Curr Opin Neurol 2008;21:195-201.

[4] Devinsky O, Gazzola D, LaFrance WC. Differentiating between nonepileptic and epileptic seizures. Nat Rev Neurol 2011;7:210-20.

[5] Ding J-R, An D, Liao W, Li J, Wu G-R, Xu Q et al. Altered functional and structural connectivity networks in psychogenic non-epileptic seizures. PLoS One 2013;8:e63850. 
[6] Van der Kruijs SJM, Bodde NMG, Vaessen MJ, Lazeron RHC, Vonck K, Boon P, et al. Functional connectivity of dissociation in patients with psychogenic non-epileptic seizures. J Neurol Neurosurg Psychiatry 2012;83:239-47.

[7] Labate A, Cerasa A, Mula M, Mumoli L, Gioia MC, Aguglia U, et al. Neuroanatomic correlates of psychogenic nonepileptic seizures: a cortical thickness and VBM study. Epilepsia 2012;53:377-85.

[8] Krishnan B, Faith A, Vlachos I, Roth A, Williams K, Noe K, et al. Resetting of brain dynamics: epileptic versus psychogenic nonepileptic seizures. Epilepsy Behav 2011;22(Suppl. 1):S74-81.

[9] Arzy S, Collette S, Wissmeyer M, Lazeyras F, Kaplan PW, Blanke O. Psychogenic amnesia and self-identity: a multimodal functional investigation. Eur J Neurol 2011;18:1422-5.

[10] Arzy S, Allali G, Brunet D, Michel CM, Kaplan PW, Seeck M. Antiepileptic drugs modify power of high EEG frequencies and their neural generators. Eur J Neurol 2010;17:1308-12.
[11] Duda R, Hart P Stork D. Pattern classification New York: NY: Wiley Interscience· 2001.

[12] Groppe DM, Bickel S, Keller CJ, Jain SK, Hwang ST, Harden C, et al. Dominant frequencies of resting human brain activity as measured by the electrocorticogram. Neuroimage 2013;79:223-33.

[13] Tóth B, Boha R, Pósfai M, Gaál ZA, Kónya A, Stam CJ, et al. EEG synchronization characteristics of functional connectivity and complex network properties of memory maintenance in the delta and theta frequency bands. Int J Psychophysiol 2012;83:399-402.

[14] Uhlhaas PJ, Singer W. Neural synchrony in brain disorders: relevance for cognitive dysfunctions and pathophysiology. Neuron 2006;52:155-68.

[15] Blanke O, Arzy S. The out-of-body experience: disturbed self-processing at the temporo-parietal junction. Neuroscientist 2005;11:16-24.

[16] Arzy S, Molnar-Szakacs I, Blanke O. Self in time: imagined self-location influences neural activity related to mental time travel. J Neurosci 2008;28:6502-7. 